\title{
Contacto con la Naturaleza: Favoreciendo la Conectividad con la Naturaleza y el Bienestar
}

\author{
Contact with Nature: Favoring Connectedness to Nature and \\ Well-being
}

\author{
Laura Pasca 10 ORCID , Juan Ignacio Aragonés ${ }^{2}$ \\ Universidad Complutense de Madrid \\ España
}

Fecha correspondencia:

Recibido: agosto 10 de 2020.

Aceptado: febrero 15 de 2021.

Forma de citar:

Pasca, L., \& Aragonés, J.I. (2021).

Contacto con la Naturaleza:

Favoreciendo la Conectividad con la

Naturaleza y el Bienestar. Rev. CES

Psico, 14(1), 100-111.

Open access

(c) Copyright

Licencia creative commons

Ética de publicaciones

Revisión por pares

Gestión por Open Journal System

DOl: http://dx.doi.org/10.21615/

cesp.14.1.8

ISSN: 2011-3080

Sobre los autores:

1. Doctora en Psicología. Profesora Facultad de Psicología, Universidad Complutense de Madrid.

Comparte

\section{Resumen}

Las personas interactúan con la Naturaleza tanto a nivel físico como cognitivo y emocional, y la investigación científica ha puesto de manifiesto la capacidad de la Naturaleza de procurar el bienestar de las personas. Con el objetivo de conocer si el contacto con la Naturaleza se relaciona con mayores niveles de conectividad con la Naturaleza como de bienestar, se realizaron dos estudios. El primero se llevó a cabo con dos muestras diferentes: una de adolescentes $(n=283)$ y otra de adultos $(n=447)$, quienes respondieron un cuestionario mediante el que se evaluó la conectividad con la Naturaleza y el bienestar. A partir de los resultados de una prueba t de Student se observó mayor conectividad con la Naturaleza y bienestar en personas con un contacto frecuente con la Naturaleza, en ambas muestras. En el segundo estudio $(n=63)$, se investigó dicha relación en personas tras realizar un recorrido por un entorno natural artificial. Los resultados del análisis de varianza muestran que las personas que realizaron el recorrido obtuvieron mayores niveles de conectividad y afecto positivo, respecto a la línea base, que quienes no vivieron esta experiencia. Este trabajo evidencia las ventajas de la intervención psicológica mediante el contacto con la Naturaleza.

Palabras clave: Naturaleza, Ambientes Naturales, Bienestar, Conectividad, Afecto Positivo, Afecto Negativo.

\section{Abstract}

Humans relate to nature at a physical, cognitive, and emotional level, and scientific research has shown how this relation impacts on people's well-being. Two studies were carried out to determine whether contact with nature is related to higher levels of connectedness and well-being; one consisted of adolescents $(n=283)$ and the other of adults $(n=447)$, who responded to a questionnaire aimed at assessing connectivity with Nature and well-being. A greater connectedness and well-being were observed in people with frequent contact with nature. In the second study $(n=63)$, this relationship was studied in people after taking a tour through an artificial natural environment. The results show that the people who took the tour had higher levels of connectedness and positive affect, with levels increasing 
2. Doctor en Psicología. Profesor y Coordinador del Máster de Psicología Social. Facultad de Psicología, Universidad Complutense de Madrid. with respect to the baseline. In this study, the advantages of psychological intervention through contact with nature are evident.

Keywords: Nature, Natural Environments, Wellbeing, Connectedness, Positive Affect, Negative Affect.

\section{Introducción}

La relación entre el ser humano y la Naturaleza es tan intensa que se le ha conferido un carácter innato, por el cual las personas sienten una necesidad biológica de mantenerse en contacto con la misma (Kellert \& Wilson, 1993). No obstante, esta relación está matizada, entre otros factores, por los diferentes contextos históricos y culturales. A medida que la sociedad occidental ha evolucionado, la Naturaleza, conformada por los entornos en los que no es evidente el impacto humano, tiene cada vez menos cabida en la cotidianeidad de las personas, debido a su tendencia a llevar un modo de vida urbano que las aleja de los ambientes naturales (Descola, 2005); situación que conlleva una separación entre el ser humano y la Naturaleza, no sólo a nivel físico, sino también cognitivo y emocional.

A través del tiempo y las diferentes culturas ha existido la creencia de que el contacto con la Naturaleza favorece la salud de las personas, es decir, que los elementos naturales como las plantas o el agua pueden reducir la ansiedad y ayudar a lidiar con el estrés del día a día (Ulrich, 1979). También la investigación científica ha puesto de manifiesto que el contacto con la Naturaleza aumenta los niveles de bienestar subjetivo (e. g. Marselle, Irvine, \& Warber, 2014), reduce el estrés (e. g. Tyrväinen et al., 2014) y mejora la cognición del ser humano (e. g. Berman, Jonides, \& Kaplan, 2008; Mayer, Frantz, Bruehlman-Senecal, \& Dolliver, 2009). Esta capacidad potencial de la Naturaleza de procurar bienestar a las personas ha promovido su relevancia en el ámbito de la investigación psicoambiental (Olivos \& Ernst, 2017).

En este sentido, la Psicología Ambiental ha realizado grandes esfuerzos por describir las relaciones, no solo físicas, sino también cognitivas y emocionales, entre el ser humano y la Naturaleza; de manera particular, se ha interesado por estudiar el modo como el contacto de las personas con la Naturaleza propicia niveles más altos de preocupación y comportamiento proambiental; ideas que constituyen la perspectiva de la conectividad con la Naturaleza (Beery \& Wolf-Watz, 2014). La conectividad se refiere, además a aspectos culturales y de evolución espiritual (Dutcher, Finley, Luloff, \& Johnson, 2007). Desde esta línea de estudio, se ha encontrado que la desconexión de los entornos naturales tiene un impacto negativo en el bienestar emocional de las personas (Capaldi, Dopko, \& Zelenski, 2014), y la conexión con estos entornos se asocia con un aumento de la felicidad (Zelenski \& Nisbet, 2014). Se ha observado que, además, el contacto con la Naturaleza conlleva un aumento de la conectividad con la misma, y esta última variable actúa como mediadora entre el contacto con la Naturaleza y el bienestar (Mayer et al., 2009).

Respecto al concepto de bienestar existen tradicionalmente dos grandes perspectivas de estudio: la hedónica, basada en el placer y en la felicidad, y la eudaimónica, relacionada con la autorrealización y una forma de vida repleta de propósitos (Deci \& Ryan, 2008). Actualmente, la perspectiva hedonista adopta el término "Bienestar Subjetivo", conformado por la satisfacción con la vida, la presencia de estados de ánimo positivos y la ausencia de negativos (Diener, 2000). La perspectiva eudaimónica, por su parte, recurre al término de "Bienestar Psicológico", fundamentado en 
Pág 102

El concepto de bienestar se asocia con frecuencia al contacto con la Naturaleza. Diversos trabajos han mostrado que pasar tiempo en contacto con la Naturaleza incrementa los niveles de bienestar tanto en adultos (e. g. Bratman, Hamilton, Hahn, Daily, \& Gross, 2015) como en niños (e. g. Carrus, Passiatore, Pirchio, \& Scopelliti, 2015). los potenciales de autorrealización de las personas, y comprende un ideal de lucha por la excelencia y la perfección que da significado y propósito a la vida (Waterman, 1993).

El estudio del "Bienestar Subjetivo" se ha guiado básicamente por dos concepciones (Ryff \& Keyes, 1995). La primera de ellas se basa en la satisfacción con la vida y el grado en que las personas perciben su vida cercana a su ideal de buena vida. La segunda concepción hace referencia al afecto tanto positivo como negativo, concretamente a una predominancia del primero frente al segundo. El afecto positivo refleja el grado en el que una persona se siente entusiasmada, activa y alerta, en un estado de alta energía, total concentración y agrado (Watson, Clark, \& Tellegen, 1988). Por su parte, el afecto negativo se refiere a un estado de angustia y desagrado, e incluye estados de ánimo como el enfado, el desprecio, el disgusto, la culpa, el miedo y el nerviosismo. Sin embargo, se ha observado que ambas dimensiones -afecto positivo y afecto negativo-son independientes, lo que ha llevado a tomar el equilibrio entre ellas como un índice de felicidad (Ryff, 1989).

Como se comentó anteriormente el concepto de bienestar se asocia con frecuencia al contacto con la Naturaleza. Diversos trabajos han mostrado que pasar tiempo en contacto con la Naturaleza incrementa los niveles de bienestar tanto en adultos (e. g. Bratman, Hamilton, Hahn, Daily, \& Gross, 2015) como en niños (e. g. Carrus, Passiatore, Pirchio, \& Scopelliti, 2015). Este bienestar puede ser tanto eudaimónico como hedónico (Aragonés, Olivos, Lima, \& Loureiro, 2013), y cada uno de ellos se asocia con diferentes formas de contacto. Así, White, Pahl, Wheeler, Depledge y Fleming (2017) observaron que las personas que acuden con mayor frecuencia a entornos naturales muestran mayores niveles de bienestar eudaimónico, mientras que las visitas puntuales a entornos naturales se asocian con el bienestar hedónico o, más concretamente, con el afecto positivo. Es decir, aquellos que visitan frecuentemente la Naturaleza sienten en mayor medida que su vida merece la pena, mientras que las personas que realizan visitas puntuales se sienten más felices en esos momentos.

En un estudio llevado a cabo por Pasca (2018) se observó que los distintos tipos de entornos -naturales, naturales artificiales y construidos- visualizados en fotografías suscitan diferentes niveles de bienestar; no obstante, esta diferencia no se encontró entre los entornos naturales y naturales artificiales, es decir, entre aquellos sin presencia humana y aquellos impregnados de la misma, a pesar de tener aspecto natural. Lo anterior sugiere que, si el bienestar de las personas no discrimina entre ambos tipos de entornos, entonces no es necesario visitar lugares naturales "puros", como por ejemplo las playas vírgenes, para experimentar elevados niveles de bienestar. Asimismo, Carrus et al. (2017) encontraron que después de visitar un jardín botánico las personas reportaron mayores niveles de bienestar. Estos hallazgos permiten pensar que se pueden aumentar los niveles de bienestar visitando lugares más accesibles para muchas personas, como los parques forestales o aquellos entornos que, aunque estén ubicados en la ciudad y hayan sido diseñados por el ser humano muestran apariencia natural.

Teniendo en cuenta lo anterior, se realizó un primer estudio con el objetivo de estudiar la relación entre la frecuencia de contacto con la Naturaleza y la conectividad con la misma y el bienestar. Se llevó a cabo con dos muestras diferentes, una formada por adolescentes y otra por adultos, ambas seleccionadas de la población general para procurar una mayor generalización de los resultados independientemente de la edad 
de las personas. En segundo lugar, se llevó a cabo otro estudio con el objetivo conocer si las personas presentan mayores niveles de conectividad con la Naturaleza, tanto a nivel cognitivo como emocional, y de bienestar, tras realizar un recorrido por un entorno natural artificial. A continuación, se describe el método y los resultados de cada uno de los estudios:

\section{ESTUDIO 1: Frecuencia de contacto con la Naturaleza, conecti- vidad con la misma y bienestar.}

En este estudio se analizó la relación entre la frecuencia de contacto con la Naturaleza, la conectividad con la misma y el bienestar.

\section{Método}

\section{Participantes}

Participaron, por un lado, 283 estudiantes de Educación Secundaria Obligatoria de tres institutos diferentes de la Comunidad de Madrid. Uno de ellos situado en el centro de la ciudad de Madrid $\left(n_{1}=99\right)$ y dos situados en la periferia $\left(n_{2}=102\right.$ y $\left.n_{3}=82\right)$. El $55,1 \%$ eran mujeres y con edades comprendidas entre 13 y 17 años, con una media de $14,59(D T=0,92)$. Por otro lado, participaron 447 personas adultas de población general urbana de la Comunidad de Madrid, de las cuales $58,4 \%$ eran mujeres, con edades comprendidas entre 18 y 77 años, con una media de 38,23 (DT = 14,14).

\section{Diseño}

Se realizó un diseño transversal, tomando una medida de las variables para cada una de las muestras en un único momento. Se trata de un diseño selectivo, ya que el objetivo del mismo fue analizar la relación entre las variables.

\section{Instrumento y procedimiento}

Se diseñó un cuestionario autoadministrado en formato papel y lápiz que comprendía, en primer lugar, un ítem acerca de la frecuencia de contacto con la Naturaleza durante los 12 meses anteriores. El formato de respuesta fue de 5 puntos, siendo 1 = nada; $2=$ de 1 a 3 veces; $3=$ de 4 a 6 veces; $4=$ de 7 a 10 veces; $5=$ más de 10 veces. En segundo lugar, se incluyó la escala PANAS (por sus siglas en ingles de Positive and Negative Affect Schedule) de Afecto Positivo (AP) y Negativo (AN) en la adaptación de López-Gómez, Hervás y Vázquez (2015), para medir el bienestar, siendo en el caso de los adolescentes el valor de alfa de Cronbach de 0,83 para el AP y 0,80 para AN y de 0,84 para AP y de 0,86 para AN, en el caso de la muestra de adultos. Dicha escala se compone de 20 ítems tipo Likert de 5 puntos, siendo cada uno de los ítems un adjetivo en el que el participante debe situarse dependiendo de cómo se sienta en ese momento. En tercer lugar, para medir el componente cognitivo del vínculo entre las personas y la Naturaleza, se incluyó la Escala de Conectividad con la Naturaleza (CNS, por sus siglas en inglés de Connectedness to Nature Scale) en su versión reducida (Pasca, Aragonés, \& Coello, 2017) ( $\alpha=0,78$ en la muestra adolescente y $\alpha=0,86$ en la muestra de adultos). Ésta consta de siete ítems tipo Likert, en los que los participantes deben situarse en un continuo de siete puntos que expresan su grado de acuerdo o desacuerdo con cada una de las afirmaciones. A continuación, para medir el componente emocional de la conexión entre las personas y la Naturaleza se utilizó la Escala de Amor y Cuidado hacia la Naturaleza (LCS, por sus siglas en inglés de Love and Care for Nature Scale) de Perkins (2010) ( $\alpha=0,95$ en la muestra adolescente y $a=0,96$ en la muestra de adultos). Ésta consta de 15 ítems tipo Likert, en los que los participantes 
deben situarse en un continuo de siete puntos que expresan su grado de acuerdo o desacuerdo con cada una de las afirmaciones. Finalmente, se incluyeron preguntas sociodemográficas (edad y sexo).

En el caso de los participantes adolescentes, tras obtener la autorización para participar por parte de sus madres y padres, el cuestionario fue cumplimentado en las aulas de los diferentes institutos durante un receso que se hizo durante la clase. Respecto a los participantes de la población general, un grupo de estudiantes de Psicología aplicaron el cuestionario a una muestra incidental teniendo en cuenta que se cumplieran unas cuotas determinadas de edad y género, con el fin de que la muestra fuera lo más equilibrada posible. En ambas muestras los participantes debieron aceptar la participación aceptando el consentimiento informado.

Para llevar a cabo la presente investigación se siguieron los principios éticos de psicólogos y código de conducta establecidos por la APA. Así, se declara conformidad con el reglamento (UE) 2016/679 del Parlamento Europeo y del Consejo de 27 de abril de 2016 de protección de datos de carácter personal.

\section{Análisis de datos}

En primer lugar, para agrupar a los participantes con base en la frecuencia de contacto, se calculó el percentil 50 en dicha variable.

Posteriormente, se llevo a cabo una prueba t de Student, tomando la variable resultante anteriormente como variable de agrupación, para cada una de las variables objeto de estudio.

\section{Resultados}

En primer lugar, en cada una de las muestras, se agruparon los participantes tomando como criterio el percentil 50 en la variable de frecuencia de contacto con la Naturaleza, obteniendo así dos tipos de participantes: aquellos con una frecuencia de contacto alta (contacto alto) y aquellos con una frecuencia de contacto baja (contacto bajo). Tomando esta variable de agrupación, se realizaron, en cada una de las muestras, cuatro pruebas $t$ de Student para muestras independientes, una para cada una de las variables criterio -CNS, LCS, Afecto Positivo (AP) y Afecto Negativo (AN)-, cuyas medias se pueden observar en la Tabla 1. Respecto a la CNS, se encontraron diferencias estadísticamente significativas entre ambos grupos tanto en la muestra de adolescentes ( $t(281)=-4,346 ; p<0,001 ; d=0,52)$, como en la muestra de adultos ( $(443)=-4,31 ; p<0,001 ; d=0,42$ ), siendo mayores las puntuaciones en el grupo con una mayor frecuencia de contacto con la Naturaleza. Y en cuanto a la LCS, se encontraron resultados similares tanto en la muestra de adolescentes $(t(281)=-5,528 ; p<0,001 ; d=0,66)$ como en la de adultos $(t(405,69)=-8,20 ; p<$ $0,001 ; d=0,80)$. Por otro lado, al analizar el bienestar se encontraron, en primer lugar, diferencias estadísticamente significativas en AP entre ambos grupos en la muestra adolescente $(t(281)=-3,282 ; p<0,01 ; d=0,39)$ y en la muestra de adultos (t $(424)=-2,08 ; p<0,01 ; d=0,20) ; y$ no se encontraron dichas diferencias en AN ni en la muestra adolescente $(\mathrm{t}(281)=-0,715 ; \mathrm{p}=0,475)$ ni en la de adultos ( $(429)=$ 0,$75 ; p=0,475$ ). 
Tabla 1. Medias, por cada muestra, en cada una de las variables criterio en base a la frecuencia de contacto.

\begin{tabular}{ccccccc}
\hline & \multicolumn{2}{c}{ Muestra adolescentes } & \multicolumn{2}{c}{ Muestra adultos } \\
\hline & Contacto bajo & & Contacto alto & Contacto bajo & & Contacto alto \\
\hline M (D. T.) & & M (D. T.) & M (D. T.) & & M (D. T.) \\
\hline CNS & $4,28(0,99)$ & $* *$ & $4,76(0,96)$ & $4,37(1,25)$ & ${ }^{* *}$ & $4,88(1,12)$ \\
\hline LCS & $4,34(1,05)$ & $* *$ & $4,96(1,02)$ & $4,66(1,17)$ & ${ }^{* *}$ & $5,50(0,95)$ \\
\hline AP & $31,43(6,94)$ & $*$ & $34,08(6,48)$ & $32,03(6,13)$ & $*$ & $32,37(6,82)$ \\
\hline AN & $15,85(5,52)$ & n. S. & $16,60(5,48)$ & $18,07(6,66)$ & n. s. & $17,58(6,68)$ \\
\hline${ }^{*} p<0,01 ;{ }^{* *} p<0,001 ;$ n. s.: diferencia estadísticamente no significativa. & & & &
\end{tabular}

\section{ESTUDIO 2: Niveles de conectividad con la Naturaleza y de bienestar, tras realizar un recorrido por un entorno natural}

Según los resultados obtenidos en el estudio 1 se observó una relación entre la frecuencia de contacto autoinformada con la Naturaleza y la conectividad con la misma, así como con el bienestar de los participantes. Para comprobar en qué medida estas relaciones se mantienen cuando las personas realizan una experiencia en un entorno natural, se llevó a cabo un segundo estudio en el que se estudiaron las mismas variables, pero en esta ocasión haciendo una comparación entre un grupo que realiza un recorrido por un entorno natural artificial y un grupo control.

\section{Método}

\section{Participantes}

Participaron 63 estudiantes de la Facultad de Psicología de la Universidad Complutense de Madrid, de los cuales $74,6 \%$ eran mujeres, con un rango de edad comprendido entre los 18 y los 27 años, siendo la media 20,11 (DT = 2,5).

\section{Diseño}

El presente estudio se realizó en dos niveles, uno intergrupo y otro de medidas repetidas. Concretamente, se llevó a cabo un diseño cuasi experimental 2 (grupo: excursión vs. cuasi control) x 2 (momento temporal: línea base vs. experiencia).

\section{Instrumento}

Se elaboró un cuestionario compuesto por la CNS en su versión reducida de 7 ítems (Pasca et al., 2017), la LCS de Perkins (2010), y la PANAS en la adaptación de López-Gómez et al. (2015). Escalas previamente descritas. Las alfas obtenidas en los 63 participantes en la línea base fueron: CNS $(\alpha=0,819)$, LCS $(\alpha=$ $0,945), \operatorname{AP}(\alpha=0,862)$ y $\operatorname{AN}(\alpha=0,815)$.

\section{Procedimiento}

En un primer momento se administró el cuestionario determinado a los 63 participantes en las aulas de la Facultad de Psicología, tras haber recibido su consentimiento informado. En un segundo momento, aproximadamente un mes después, se conformaron dos grupos: el denominado "grupo excursión" con quienes se presentaron como voluntarios a realizar un recorrido por un entorno natural artificial y el resto constituyó el grupo cuasi control. El grupo excursión fue llevado al Parque Forestal Adolfo Suarez ${ }^{1}$, situado a 2,3 km de la Facultad de Psicología, donde realizaron un 
recorrido autoguiado establecido previamente por los investigadores y señalado en un mapa del parque. Los participantes fueron recogidos en grupos de seis personas en la sede de la Facultad de Psicología, y llevados al Parque Forestal, siendo éste un trayecto de 8 minutos. Una vez allí, se les entregó el mapa y comenzaron el recorrido, de uno en uno, con diez minutos de diferencia, ya que debían realizarlo en solitario para evitar el efecto de la interacción social. Se les dio la instrucción de que enviaran comentarios y fotografías del recorrido a través del teléfono móvil a un número que se les informó con anterioridad, con el fin de que prestaran atención a los elementos del entorno. Al final del recorrido, antes de abandonar el parque, una de las investigadoras les entregó para su cumplimentación el mismo cuestionario aplicado en el primer momento. El grupo cuasi control respondió al mismo cuestionario en las aulas de la Facultad de Psicología un día antes a la realización del recorrido por el grupo excursión.

Para llevar a cabo la presente investigación (ambos estudios) se siguieron los principios éticos de psicólogos y código de conducta establecidos por la APA. Así, se declara conformidad con el reglamento (UE) 2016/679 del Parlamento Europeo y del Consejo de 27 de abril de 2016 de protección de datos de carácter personal.

\section{Análisis de datos}

Para llevar a cabo el objetivo, se realizó un análisis de varianza de dos factores de medidas repetidas en uno de ellos, siendo el factor intersujetos el grupo (excursión vs. cuasi control) y el factor intrasujeto el momento temporal (pre-post).

\section{Resultados}

La conectividad -CNS y LCS- y el bienestar -AP y AN- fueron analizados a partir del ajuste de un ANOVA de dos factores con medidas repetidas en uno de ellos, para cada una de las variables dependientes.

En primer lugar, se tomó como variable dependiente la puntuación en la CNS. Tanto para el factor intergrupo -cuasi control vs excursión- $(F(1,61)=7,09 ; p<0,01$; = $0,02)$, como para el factor intragrupal momento temporal - línea base vs. experiencia - $(F(1,61)=6,37 ; p<0,05 ;=0,02)$, como para el efecto interacción entre ambos $(F(1,61)=7,15 ; p<0,01 ;=0,02)$ se encontró un efecto estadísticamente significativo. Los resultados de la interacción entre las dos variables se pueden observar en la Figura 1, siendo la diferencia entre los efectos simples estadísticamente significativa $(t(61)=2,674 ; p<0,01)$. En referencia a los efectos simples de la variable cuasi control-excursión, se encontraron diferencias estadísticamente significativas entre el grupo cuasi control $(M=4,32 ; D T=0,19)$ y el grupo excursión $(M=5,29 ; D T=0,20)$ en el segundo momento, siendo mayor la conectividad con la Naturaleza en el grupo excursión; mientras que en la línea base no se presentaron tales diferencias entre los grupos. En cuanto a los efectos simples de la variable temporal, se encontraron diferencias significativas entre la línea base $(M=4,71 ; D T=0,20)$ y la segunda medida en el grupo excursión $(M=5,29 ; D T=0,20)$, siendo mayor la conectividad con la Naturaleza; mientras que no se presentaron diferencias estadísticamente significativas en el grupo cuasi control, mostrando las mismas puntuaciones en ambos momentos. 


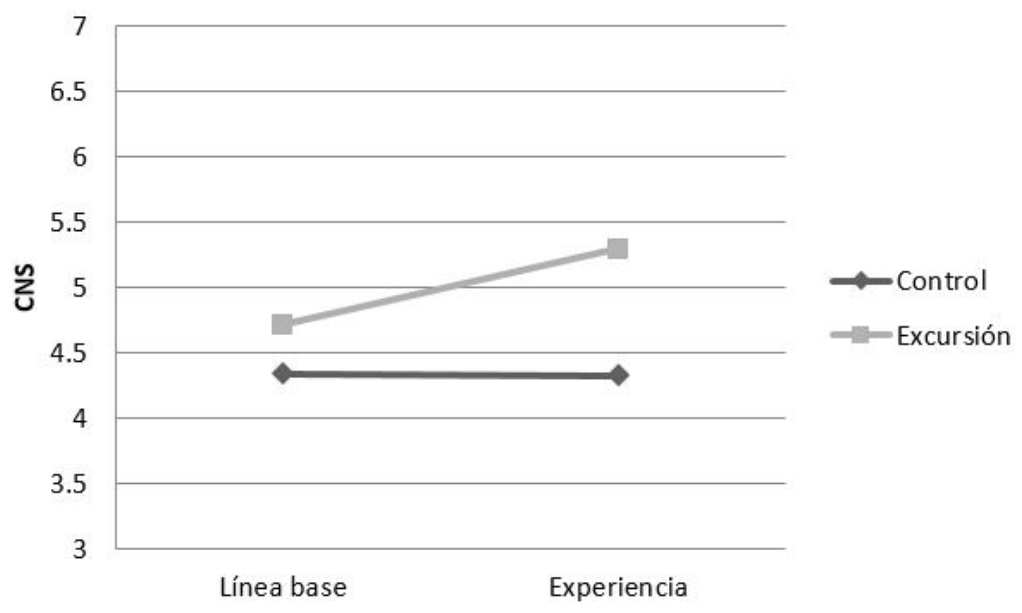

Figura 1. Interacción experiencia*momento en CNS.

En segundo lugar, se tomó como variable dependiente la puntuación obtenida por los participantes en la LCS. Tanto para el factor experiencia -excursión vs cuasi control- $(F(1,61)=4,37 ; p<0,05 ;=0,01)$, como para el factor momento temporal $(F(1,61)$ $=8,40 ; \mathrm{p}<0,01 ;=0,03)$, no se encontró un efecto estadísticamente significativo, $\mathrm{y}$ no se presentó tal efecto de la interacción entre ambos $(F(1,61)=1,74 ; p=0,19)$. En cuanto al momento temporal, las puntuaciones de la LCS presentaron diferencias estadísticamente significativas $(p<0,01)$ entre la media obtenida en la línea base ( $M$ $=4,88 ; D T=0,13)$ y la obtenida en la segunda medida $(M=5,10 ; D T=0,13)$, siendo superior esta última. Igual ocurre con los efectos principales de la condición, siendo mayor la media en amor y cuidado hacia la Naturaleza en el grupo experimental ( $M$ $=5,25 ; D T=0,18)$ que en el grupo cuasi control $(M=4,72 ; D T=0,17)$.

En cuanto al bienestar, en primer lugar, se analizó el afecto positivo. Tanto para el factor experiencia -excursión vs cuasi control- $(F(1,61)=9,23 ; p<0,01 ;=0,03)$, como para el efecto interacción entre ambos $(F(1,61)=8,19 ; p<0,01 ;=0,03)$ se encontró un efecto estadísticamente significativo. Sin embargo, no se halló tal efecto del factor momento temporal -línea base vs experiencia- $(F(1,61)=0,36 ; p=0,55)$.

El efecto de la interacción se puede observar gráficamente en la Figura 2, cuyo efecto es estadísticamente significativo ( $\mathrm{t}(61)=2,861 ; \mathrm{p}<0,01)$. El análisis de los efectos simples de la condición muestra que en la línea base no existen diferencias estadísticamente significativas entre el grupo cuasi control $(M=31,35 ; D T=1,15)$ y el grupo excursión ( $M=32,90 ; D T=1,24)$; mientras que en el segundo momento sí se encuentran tales diferencias $\left(M_{\text {cuasi control }}=29,21(D T=1,15)\right.$ y $\left.M_{\text {excursión }}=36,17(D T=1,24)\right)$. 


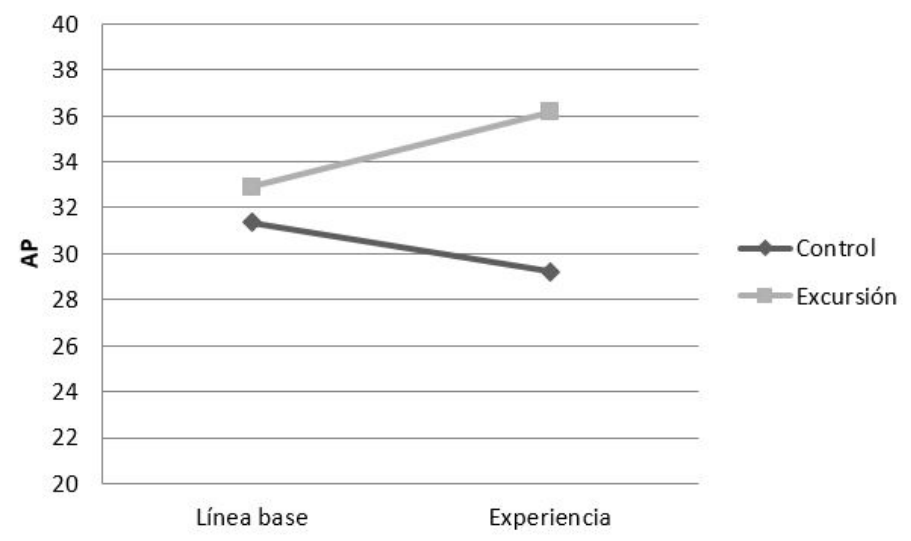

Figura 2. Interacción en el afecto positivo.

Por último, se llevó a cabo este mismo análisis tomando como variable dependiente el AN. Para el factor intregrupo -cuasi control vs excursión- existe un efecto estadísticamente significativo $(F(1,61)=5,81 ; p<0,05 ;=0,02)$, no se presentó tal efecto del factor momento temporal $(F(1,61)=0,55 ; p=0,46)$, ni de la interacción entre ambos $(F(1,61)=1,46 ; p=0,23)$. Si se analizan los efectos principales, se comprueba que el afecto negativo es mayor en el grupo cuasi control $(M=17,49 ; D T=0,93)$ que en el grupo experimental $(M=14,17 ; D T=1,01)$.

\section{Discusión}

Con el objetivo de conocer si el contacto con la Naturaleza se relaciona con mayores niveles de conectividad con la Naturaleza como de bienestar, se realizaron dos estudios. Los resultados del primer estudio muestran como las personas que mantienen un contacto frecuente con la Naturaleza, sean adolescentes o adultos, se sienten más conectados con la misma, tanto a nivel afectivo como cognitivo; además, muestran mayores niveles de afecto positivo y no presentan diferencias en cuanto al afecto negativo comparadas con quienes tienen menos contacto con la Naturaleza.

No obstante, estos resultados se obtuvieron a través de una medida autoinformada de la frecuencia de contacto con la Naturaleza, por tanto, sometida a la subjetividad. Esta evaluación realizada a partir de recuerdos sobre la conducta es más fiable que aquellas que se basa en opiniones, aunque no deja de estar sometida a sesgos, como ya observaba Greenwald (1980). Quizá operativizaciones más observacionales permitan una mayor precisión de la relación.

Para poner a prueba en qué medida estas relaciones se mantienen o se intensifican cuando las personas realizan una experiencia en un entorno natural, se llevó a cabo un segundo estudio. Los resultados muestran que al comparar un grupo de participantes tras realizar un recorrido por un parque forestal frente a un grupo cuasi control, los primeros obtienen puntuaciones estadísticamente más altas que los segundos tanto en conectividad con la Naturaleza como en bienestar. Hallazgos similares a los reportados por Schnell, Harel y Mishori (2019), quienes encontraron que las personas más conectadas con la Naturaleza mostraron mayores niveles de bienestar, explicando, de hecho, la primera variable un $49 \%$ de la varianza de la segunda. En esta misma línea, Mena-García, Olivos, Loureiro y Navarro (2019) encontraron que las personas que realizaron un recorrido por un entorno natural se mostraron más conectadas con la Naturaleza, además de evocar mayores emociones positivas. 
El contacto con la Naturaleza fomenta el afecto positivo de las personas (entusiasmo, activación, concentración y agrado), así como su conectividad con la Naturaleza tanto en aquellas que frecuentemente visitan y se ponen en contacto con la Naturaleza, como en quienes lo hacen de forma puntual o más esporádica, aunque en este último caso el efecto sólo se observa tras la experiencia.
Por otro lado, al comparar las puntuaciones del grupo de participantes que realiza un recorrido por un entorno natural de la línea base en conectividad con la Naturaleza, medida un mes antes a la experiencia natural, y la medida inmediatamente después de la misma, se observa que aumentan tras el contacto. Similares resultados se encuentran al realizar los análisis sobre el componente emocional de la conectividad, observándose mayores vínculos con la Naturaleza que antes de vivir la experiencia del recorrido.

Respecto al efecto del contacto con la Naturaleza en el bienestar, los resultados muestran mayor afecto positivo y menor afecto negativo en las personas que realizan un recorrido por un entorno natural artificial en relación con quienes no viven esta experiencia. Sin embargo, respecto al afecto negativo, se observa que no existen diferencias estadísticamente significativas en cuanto al momento temporal, es decir, que las diferencias encontradas tras el contacto con la Naturaleza podrían no deberse al efecto de la excursión, puesto que ya existían en la línea base. Por tanto, el hecho de que las personas que han realizado la experiencia muestren menores niveles de afecto negativo que los que no la han realizado, puede obedecer a un estado previo de los participantes, el cual podría estar implicado en la formación de los grupos, es decir, que las personas con un mayor afecto negativo no se muestren motivados a prestarse como voluntarios para realizar el recorrido. Estos resultados concuerdan con los obtenidos por Mayer et al. (2009), quienes realizaron un estudio similar en el que compararon los niveles de afecto positivo y afecto negativo de un grupo de personas tras realizar una excursión por la Naturaleza frente a un grupo de personas que realizó un recorrido por la ciudad. Encontraron que las personas del primer grupo mostraron mayores niveles de afecto positivo, sin embargo, no mostraron diferencias en cuanto al afecto negativo. Adicionalmente, cabe comentar que la experiencia de contacto con la Naturaleza, en el presente estudio, fue realizada en otoño, una época del año en la que comienzan las altas temperaturas; por lo cual los resultados expuestos apoyan la idea de Brooks, Ottley, Arbuthnott y Sevigny (2017), respecto a que, a pesar de las incomodidades del frio, los efectos positivos de la Naturaleza se extienden más allá del verano.

Por tanto, el contacto con la Naturaleza fomenta el afecto positivo de las personas (entusiasmo, activación, concentración y agrado), así como su conectividad con la Naturaleza tanto en aquellas que frecuentemente visitan y se ponen en contacto con la Naturaleza, como en quienes lo hacen de forma puntual o más esporádica, aunque en este último caso el efecto sólo se observa tras la experiencia. Así, los resultados del presente trabajo ponen de manifiesto la necesidad de fomentar el contacto de las personas con la Naturaleza, ya que supondría un beneficio en el bienestar de las mismas y la posibilidad de que presenten niveles más altos de preocupación por cuidar la naturaleza y comportamientos proambientales.

\section{Agradecimientos}

Este estudio fue llevado a cabo como parte del proyecto PSI2013-41601-P, financiado por el Ministerio de Ciencia e Innovación del gobierno de España. 


\section{Referencias}

Aragonés, J. I., Olivos, P., Lima, M. L., \& Loureiro, A. (2013, junio). Connectedness, wellbeing and nature. Sesión de posters presentado en 22 Conference IAPS, Glasgow.

Beery, T. H., \& Wolf-Watz, D. (2014). Nature to place: Rethinking the environmental connectedness perspective. Journal of Environmental Psychology, 40, 198-205.

Berman, M. G., Jonides, J., \& Kaplan, S. (2008). The cognitive benefits of interacting with nature. Psychological Science, 19, 1207-1212. https://doi.org/10.1111/j.14679280.2008.02225.x

Bratman, G. N., Hamilton, J. P., Hahn, K. S., Daily, G. C., \& Gross, J. J. (2015). Nature experience reduces rumination and subgenual prefrontal cortex activation. Proceedings of The National Academy of Sciences, 112(28), 8567-8572. https://doi. org/10.1073/pnas.1510459112

Brooks, A. M., Ottley, K. M., Arbuthnott, K. D., \& Sevigny, P. (2017). Nature-related mood effects: Season and type of nature contact. Journal of Environmental Psychology, 54, 91-102. https://doi.org/10.1016/j.jenvp.2017.10.004

Capaldi, C. A., Dopko, R. L., \& Zelenski, J. M. (2014). The relationship between nature connectedness and happiness: a meta-analysis. Frontiers in Psychology, 5, 976. https://doi.org/10.3389/fpsyg.2014.00976

Carrus, G., Passiatore, Y., Pirchio, S., \& Scopelliti, M. (2015). Contact with nature in educational settings might help cognitive functioning and promote positive social behaviour Psyecology, 6(2), 191-212. https://doi.org/10.1080/21711976.2015.1 $\underline{026079}$

Carrus, G., Scopelliti, M., Panno, A., Lafortezza, R., Colangelo, G., Pirchio, S., ... Semenzato, P. (2017). A Different Way to Stay in Touch with 'Urban Nature': The Perceived Restorative Qualities of Botanical Gardens. Frontiers in Psychology, 8 , 914. https://doi.org/10.3389/fpsyg.2017.00914

Deci, E. L., \& Ryan, R. M. (2008). Hedonia, eudaimonia, and well-being: An introduction. Journal of Happiness Studies, 9, 1-11. https://doi.org/10.1007/s10902-0069018-1

Descola, P. (2005). Par-delà la nature et culture [Entre la Naturaleza y Cultura]. Paris: Gallimard.

Diener, E. (2000). Subjective well-being: The science of happiness and a proposal for a national indicator. American Psychologist, 55, 34-43. https://doi. org/10.1037//0003-066X.55.1.34

Dutcher, D. D., Finley, J. C., Luloff, A. E., \& Johnson, J. B. (2007). Connectivity with Nature as a Measure of Environmental Values. Environment and Behavior, 39, 474-493. https://doi.org/10.1177/0013916506298794

Greenwald, A. G. (1980). The Totalitarian Ego Fabrication and Revision of Personal History. American Psychologist, 35, 603-618.

Kellert, S. R., \& Wilson, E.O. (Eds.) (1993). The Biophilia Hypothesis. Washington DC: Island Press.

López-Gómez, I., Hervás, G., \& Vázquez, C. (2015). Adaptación de las "escalas de afecto positivo y negativo» (PANAS) en una muestra general española. Psicología Conductual, 23, 529.

Marselle, M. R., Irvine, K. N., \& Warber, S. L. (2014). Examining group walks in nature and multiple aspects of well-being: A large-scale study. Ecopsychology, 6, 134147. https://doi.org/10.1089/eco.2014.0027

Mayer, F. S., Frantz, C., Bruehlman-Senecal, E., \& Dolliver, K. (2009). Why Is Nature Beneficial?: The Role of Connectedness to Nature. Environment and Behavior, 41, 607-643. https://doi.org/10.1177/0013916508319745 
Mena-García, A., Olivos, P., Loureiro, A., \& Navarro, O. (2019). Effects of contact with nature on connectedness, environmental identity and evoked contents. Psyecology, 11(1), 21-36. https://doi.org/10.1080/21711976.2019.1643663

Olivos, P., \& Ernst, R. (2018). Feel good or be happy. Distinctions between emotions and psychology research of wellbeing. En N. J. L. Brown, T. Lomas y F. J. Eiroa-Orosa (Eds.), International Handbook of Critical Positive Psychology-a Synthesis for Social Change. (pp. 546-564). London: Routledge.

Pasca, L. (2018). Naturaleza, Conectividad y Bienestar (Tesis doctoral). Universidad Complutense de Madrid.

Pasca, L., Aragonés, J. I., \& Coello, M. T. (2017). An Analysis of the Connectedness to Nature Scale Based on Item Response Theory. Frontiers in psychology, 8, 1330. https://doi.org/10.3389/fpsyg.2017.01330

Perkins, H. E. (2010). Measuring love and care for nature. Journal of Environmental Psychology, 30, 455-463. https://doi.org/10.1016/j.jenvp.2010.05.004

Ryff, C. D. (1989). Happiness is everything, or is it? Explorations on the meaning of psychological well-being. Journal of Personality and Social Psychology, 57, 1069. https://doi.org/0022-3514/89/S00.75

Ryff, C. D., \& Keyes, C. L. M. (1995). The structure of psychological well-being revisited. Journal of Personality and Social Psychology, 69, 719. https://doi.org/0022$3514 / 95 / 53.00$

Schnell, I., Harel, N., \& Mishori, D. (2019). The benefits of discrete visits in urban parks. Urban Forestry \& Urban Greening, 41, 179-184. https://doi.org/10.1016/j. ufug.2019.03.019

Tyrväinen, L., Ojala, A., Korpela, K., Lanki, T., Tsunetsugu, Y., \& Kagawa, T. (2014). The influence of urban green environments on stress relief measures: A field experiment. Journal of Environmental Psychology, 38, 1-9. https://doi.org/10.1016/j.jenvp.2013.12.005

Ulrich, R. S. (1979). Visual landscapes and psychological well-being. Landscape research, 4, 17-23. https://doi.org/10.1080/01426397908705892

Ulrich, R. (1984). View through a window may influence recovery. Science, 224, 224225. https://doi.org/10.1126/science.6143402

Waterman, A. S. (1993). Two conceptions of happiness: Contrasts of personal expressiveness (eudaimonia) and hedonic enjoyment. Journal of Personality and Social Psychology, 64, 678. https://doi.org/0022-3514/93/S3.00

Watson, D., Clark, L. A., \& Tellegen, A. (1988). Development and validation of brief measures of positive and negative affect: the PANAS scales. Journal of Personality and Social Psychology, 54, 1063. https://doi.org/G022-3514/88/S00.75

White, M. P., Pahl, S., Wheeler, B. W., Depledge, M. H., \& Fleming, L. E. (2017). Natural environments and subjective wellbeing: Different types of exposure are associated with different aspects of wellbeing. Health \& Place, 45, 77-84. https://doi. org/10.1016/j.healthplace.2017.03.008

Zelenski, J. M., \& Nisbet, E. K. (2014). Happiness and feeling connected: The distinct role of nature relatedness. Environment and Behavior, 46, 3-23. https://doi. org/10.1177/0013916512451901 